\title{
Which Reaches the Bottom First?
}

\author{
Jordi Solbes, University of Valencia, Valencia, Spain \\ Francisco Tarin, IES L'Om, Picassent, Valencia, Spain
}

THE PHYSICS TEACHER Vol. 46, 494-485 (2008)

well-known classroom demonstration
involves the rolling of hollow and solid
objects down an incline. ${ }^{1}$ The fact that the objects roll at different rates can be used as a starting point in introducing students to rotational dynamics and rotational kinetic energy. In this paper we describe a simple quantitative version of the demonstration that is suitable for use as a laboratory experiment.

We begin by posing the following problem to our students: A solid sphere, a hollow cylinder, and a solid cylinder, all having the same mass $M$ and radius $R$, are placed at the top of an incline having angle $\theta$. If the three objects are released from rest at the same instant and all roll down without slipping, which will reach the bottom first? After the students have made their predictions, they do the demonstration for themselves and observe that the sphere reaches the bottom first, then the solid cylinder, and then the hollow one.

We next ask the students to determine, theoretically, the acceleration of each object. They quickly realize that in the absence of friction all three would accelerate at the same rate, $a=g \sin \theta$, which is contrary to their observations. Therefore, an additional force - one that depends on the shape of the objectmust be introduced. Since the objects roll rather than slide, they must experience a force of static friction, $F_{s}$. Therefore, the equation for the linear motion of the center of mass is

$$
M g \sin \theta-F_{\mathrm{s}}=M a,
$$

while the rotation about the center of mass (we have already introduced the concepts of torque and moment of inertia) is governed by

$$
F_{\mathrm{S}} R=I \alpha,
$$

where $I$ is the moment of inertia and $\alpha$ is the angular acceleration. If the body rolls without sliding, $\alpha$ $=a / R$, and from these equations we obtain

$$
a=M g \sin \theta /\left(M+\mathrm{I} / R^{2}\right) .
$$

This shows that the acceleration depends on the moment of inertia. Substituting the expressions for the moment of inertia of a solid sphere $[I=$ $\left.(2 / 5) M R^{2}\right]$, a solid cylinder $\left(I=M R^{2} / 2\right)$, and a hollow one $\left(I=M R^{2}\right)$, we find that the accelerations are $a=(5 / 7) g \sin \theta, a=2 / 3 g \sin \theta$, and $a=1 / 2 g \sin \theta$, respectively.

Then we ask our students to do an experiment to check the predicted results. They use an inclined plane with a motion sensor positioned at its upper end, as shown in Fig. 1. They roll the objects, one at a time, down the incline $\left(\theta=5^{\circ}\right)$ and set up the motion sensor to measure the position and velocity as functions of time. They plot velocity versus time and fit a straight line to the data. The slope of the line is the measured acceleration. Typical results are shown in Table I. The agreement between measured and theoretical values is generally very good, and students quickly understand that the sphere must reach the bottom first because its acceleration is the largest. 


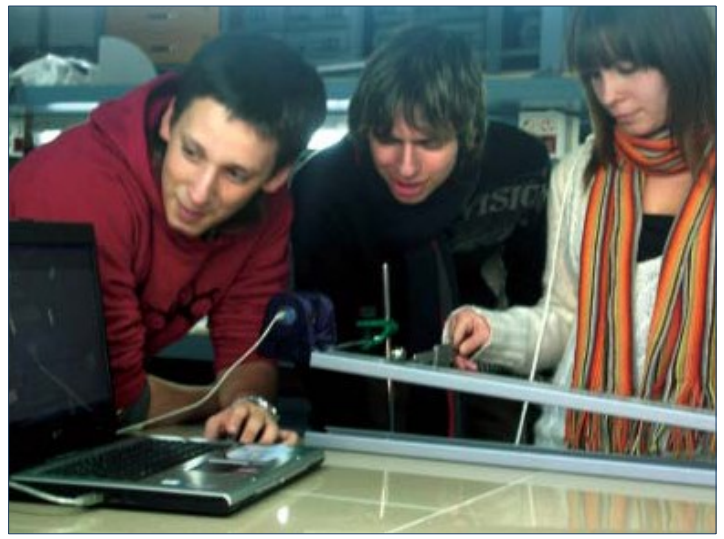

Fig. 1. Experimental setup.

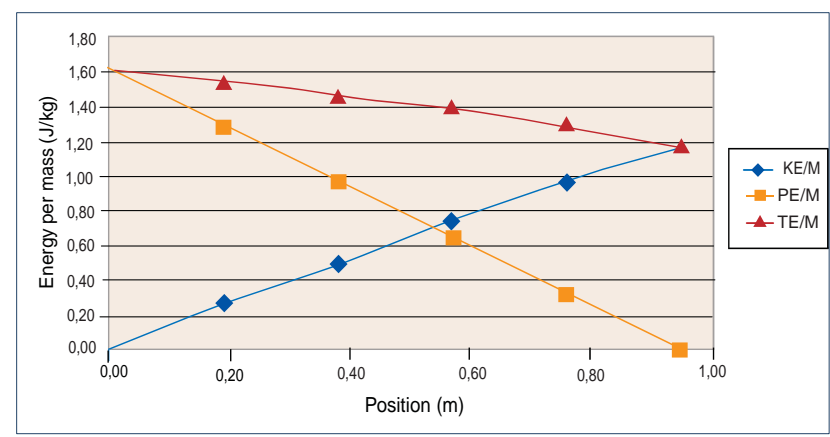

Fig. 2. Energy plots for a solid sphere rolling down a $10^{\circ}$ incline. Because rotational kinetic energy is not included, the total mechanical energy seems to decrease.

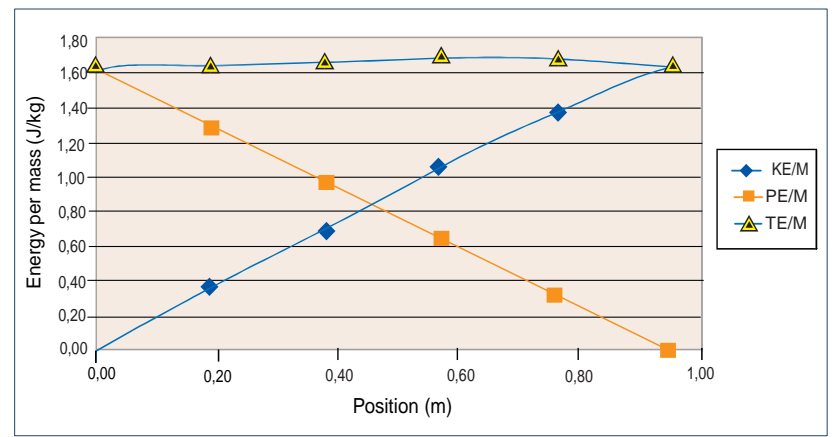

Fig. 3. Energy plots for the solid sphere on an incline. Rotational kinetic energy is included, and the total mechanical energy remains nearly constant.

The same experimental setup can be used to investigate the law of mechanical energy conservation in the case of an object rolling down an inclined plane. The potential energy is given by $P E=M g h$, where $h=$ $s \sin \theta$ and $s$ is the displacement of the object measured from the bottom of the incline. We ask our students to roll one of the objects down the incline and produce a plot of $M g h+M v^{2} / 2$ versus position as the object rolls. Many are at first surprised by the results (see Fig.
Table 1. Predicted and measured acceleration for objects rolling down an incline.

\begin{tabular}{|l|c|c|}
\hline Object & $\begin{array}{c}\text { Predicted } \\
\text { acceleration }\left(\mathrm{m} / \mathrm{s}^{2}\right)\end{array}$ & $\begin{array}{c}\text { Measured } \\
\text { acceleration }\left(\mathrm{m} / \mathrm{s}^{2}\right)\end{array}$ \\
\hline sphere & 0.61 & 0.65 \\
\hline solid cylinder & 0.57 & 0.59 \\
\hline hollow cylinder & 0.43 & 0.41 \\
\hline
\end{tabular}

2) —it appears that mechanical energy is not conserved, i.e., the kinetic energy (naively assumed to be $M v^{2} / 2$ ) does not increase at the same rate as the potential energy decreases. We guide them to the conclusion that the model they are using is too simple - that because the object is undergoing rotational as well as translational motion, the correct expression for its kinetic energy is actually $K E=M v^{2} / 2+$ $K E_{\text {rot }}$, where $K E_{\text {rot }}=I \omega^{2} / 2$. The angular speed $\omega$ is equal to $v / R$. In the case of a solid sphere, $I=2 M R^{2} / 5$ and so $K E_{\text {rot }}$ $=M v^{2} / 5$. Therefore, $K E=0.7 M v^{2}$. When students use this new expression for the kinetic energy, they obtain plots like those in Fig. 3, which show that the total mechanical energy is, in fact, nearly constant over the whole range of motion.

Our students have found this quantitative laboratory exercise to be interesting and thought provoking. We believe that they learned a great deal from carrying it out.

\section{References}

1. A number of commercial setups are available. See, for example, the PASCO Rotational Inertia Set (ME-9774) at http:// www.pasco.com, and the Ring \& Disk Moment of Inertia Demonstrator (CP75255-00) at http://www.sargent-welch. com.

PACS Codes: $01.40 \mathrm{Fk}$, 01.50.Pa

Jordi Solbes received his PhD from the University of Valencia. He currently teaches high school physics at IES Rodrigo Botet, Manises, Valencia, Spain and didactics of physics at the University of Valencia. His research interest is in physics education.

Department of Didactics of Experimental and Social Sciences, University of Valencia, Alcalde Reig, 8; 46006 València (Spain); jordi.solbes@uv.es

Francisco Tarín teaches physics in a Spanish secondary school. He received his doctorate from the University of Valencia. IES L'Om, Picassent, Valencia, Spain; ftarinma@ono.com 